\title{
Integration of Wireless Sensor Network and Web Services
}

\author{
Akbar Ghobakhlou, Alexander Kmoch and Philip Sallis \\ Geoinformatics Research Centre, \\ AUT University, Auckland New Zealand \\ Email: Akbar@aut.ac.nz
}

\begin{abstract}
Wireless Sensor Networks (WSNs) integrated with web services are becoming common in widespread applications across the world. WSNs are developed in different application domains of sensor and user types, with each typically relying on its own metadata semantics, data format and software. There is a high demand for standardising access to sensor data via internet without having to use some complex and unknown protocol. Thus, Service Oriented Architecture (SOA) is one of the key paradigms that enables the deployment of services at large-scale over the internet domain and its integration with WSNs could open new pathways for novel applications and research. The sensor web enablement initiative (SWE) within the Open Geospatial Consortium (OGC) has released a set of open standards for interoperable interface specifications and (meta) data encodings for the real time integration of sensors and sensor networks into a web services architecture. This paper describes integration of WSNs into a SOA by proposing a web service proxy linkage of the low level sensor platform to the high level SWE sensorweb architecture to treat sensors in an interoperable, platform-independent and uniform way.
\end{abstract}

Keywords: Wireless Sensor Networks, Vineyard monitoring, Service-Oriented Architecture 


\section{INTRODUCTION}

Wireless Sensor Networks (WSNs) have been gaining growing attention due to their enormous potential in providing novel solutions to numerous applications. Many of these applications need to transfer the collected sensor data to applications that are usually residing on a traditional network infrastructure such as LAN or enterprise network. An area of interest for WSN is the large-scale environmental monitoring applications. WSNs are capable of sensing, collecting and monitoring of data in real-time and can be accessed by the end users. In precision agriculture, WSNs enable monitoring and management of a large set of environmental data such as climatic, atmospheric, plant and soil parameters that influence cropland growing environments. Real-time sensor data collection is used for accurate illustrations of current conditions while forecasting future conditions and risks. The implementation WSN combined with communication networks becoming affordable and reliable to measure the agro-meteorological and crop parameters in precision agriculture (Díaz et al., 2011; Zhang et al., 2011).

The work presented in this paper is related to a research programme at Geoinformatics Research Centre (http://www.geo-informatics.org). It comprises number of projects, including building environmental data monitoring instrument set with associated data acquisition, monitoring, information modeling and visualization. This paper describes integration of WSNs into a Service Orientated Architecture (SOA) by proposing a web service proxy linkage of the low level sensor platform to the high level Sensor Web Enablement (SWE) sensorweb architecture. This approach is based on Open Geospatial Consortium's (OGC) SWE standards enabling flexible data sharing, platform-independent and uniform.

\section{WIRELESS SENSOR NETWORK}

A WSN usually has little or no infrastructure. It consists of a number of sensor nodes (few tens to thousands) and can work together to monitor a region to obtain data about the environment. There are two types of WSNs: structured and unstructured. Unstructured WSN contains dense collection of sensor nodes and often deployed in ad-hoc manner in the field. In an ad-hoc deployment, sensor nodes maybe dropped from a plane and randomly into the target area. In structured WSN on the other hand, all or some of the sensor nodes are deployed in a pre-determined locations. Structured network has fewer nodes and can be deployed with lower network maintenance and management costs (Deborah et al., 1999).

Sensor node devices are energy-limited and application-specific and therefore the power management is essential for effective network operation. The network can also communicate to the outside world by means of GPRS. For situations with difficult wireless connectivity such as mines each sensor device can store large number of sensor measurements in its SD card memory.

\subsection{System Architecture}

The proposed system architecture in this study is based upon previous work on environmental monitoring system described in (Ghobakhlou et al. 2010). It consists of nodes operating in mesh topology and deployed in various locations of interest within the crop field (vineyard in this case). Nodes include sensors, transmitters and repeaters for logging various environmental parameters.

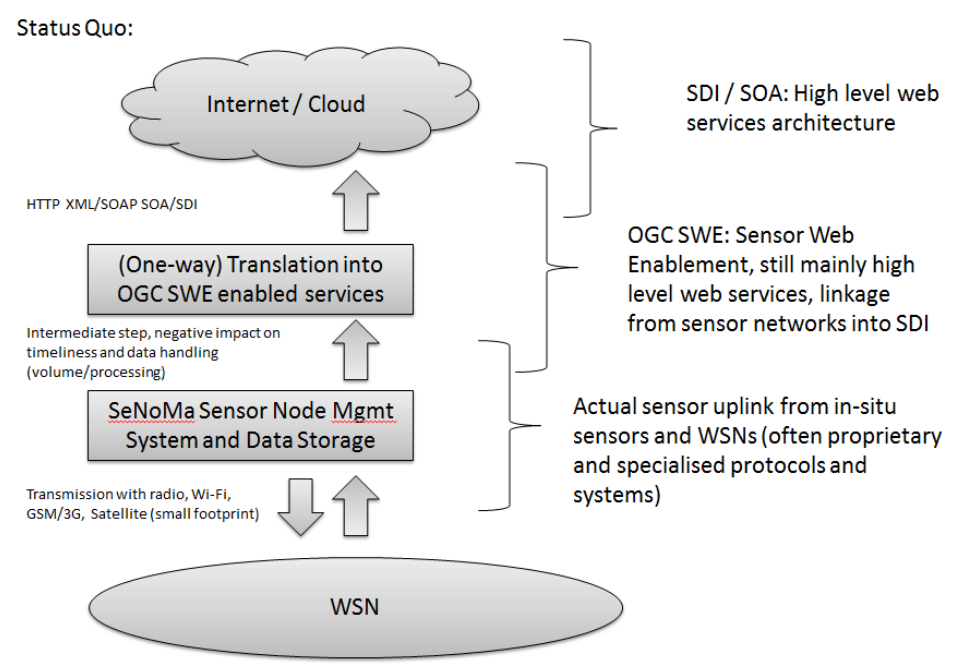

Figure 1: Status Quo of Centrally Operated Wireless Sensor Networks 
Sensor Node Management (SeNoMa) software was designed to manage WSN configuration including assigning sensors to nodes, determining logging and transmitting intervals. The WSN designed and built for this research has been mainly focused on collecting, logging and processing near-ground truth data.

Figure 1 illustrates the Status Quo of centrally operated WSN. The configuration used for implementing this WSN architecture described in more details in (Ghobakhlou et al. 2011).

\section{SERVICE ORIENTED ARCHITECTURE}

In a Service Oriented Architecture (SOA) available services, which could be data provider or processing algorithms or something else are so called "loosely coupled" (Erickson and Siau, 2008). That means by using SOAP and its Web Service Description Language (WSDL) the service descriptions/capabilities are available at runtime and can be evaluated on demand. The systems do not need to know all members initially. By relying on a predefined set of standards and service interface descriptions, the system becomes flexible to add new services to the orchestration. This concept comes from the field of business informatics but has been successfully applied to geoinformatics, too (Granell et al., 2010). Such web services form the foundation of the so called "cloud" computing.

The Open Geospatial Consortium (OGC) provides a set of standards that comply to such a desired architecture. The OGC was founded in 1994 and "is an international industry consortium of 482 companies, government agencies and universities participating in a consensus process to develop publicly available interface standards" (OGC, 2013). For the domain of linking wireless sensor networks into a service oriented architecture the OGC defines a set of service interfaces and data encodings within the Sensor Web Enablement initiative (SWE) (Botts et al., 2008). Main interfaces for data transmission and interaction with sensors and actors are the Sensor Observation Service (SOS) and the Sensor Planning Service (SPS) (OGC, 2012). "The Sensor Web Enablement (SWE) standards of the Open Geospatial Consortium (OGC) comprise the only truly open international standards suite that provides a comprehensive platform for publishing, discovering, assessing, accessing and using sensors and sensor systems of all kinds"1. Message encoding within SWE services is defined in the specifications for OWS Commons (OGC Web Service Commons), SWE Common Data Model Encoding (OGC, 2011b), Observations \& Measurements (O\&M) (OGC, 2011a) and SensorML (OGC, 2007). The application, use and evolution of the sensor web standards has been exhaustively described in Bröring et al. (2011). O\&M is a generic flexible application schema for the encoding of observation data and time-series, whereas sensor descriptions, configurations and capabilities can be expressed in SensorML.

However, OGC SWE semantics heavily rely on XML-based web-services (Simple Object Access Protocol SOAP), which induce data volume overhead through the XML mark-up (which is still not effective data) and subsequently could cause data transfer implications on low-bandwidth unreliable network connections. Alternative sensor uplink methods are proposed by Hunkeler, Lombriser, Truong and Weiss (2013). They present an "Intelligent, Manageable, Power-Efficient and Reliable Internetworking Architecture (IMPERIA). It is a centrally controlled architecture that vertically integrates a Wireless Sensor Network's (WSN) network stack with the publish/subscribe messaging middleware MQTT-S”. Also Chen, Díaz, Rubio and Troya (2013) discuss MQTT as a suitable bi-directional transport protocol for wireless sensor networks following the message queue pattern.

\section{METHODS AND MATERIALS}

To enable SeNoMa to operate in a cloud environment for ubiquitous and standardised access we need to "web enable" the WSN. However, with the XML-based overhead of the OGC SWE protocols and encodings, it is inappropriate for the low-level uplink from the WSN to the Sensor Web. This is due to two factors:

1) Available bandwidth with satellite connection, reception of directional radio or GSM/3G coverage might be limited, patchy or otherwise unreliable. Verbatim OGC SWE HTTP/SOAP XML two-way communication might be interrupted sporadically, but provides no means for recovery and therefore in next transmission cycle the full communication process needs to be re-initiated.

2) Although the XML schema provides all means to verify and validate communication and encoding, most of the transmitted data is redundant mark-up, but not environmental measurements. Yet,

\footnotetext{
${ }^{1}$ OGC Sensor Web Enablement (SWE) Homepage: http://www.ogcnetwork.net/swe
} 
transmission of data is the most energy consuming operation for an energy autarchic WSN in the field and needs to be minimised.

Therefore we propose the interlinking of OGC SWE semantics for sensor descriptions and observations (OGC Sensor Observation Service, SOS), sensor configuration and planning (OGC Sensor Planning Service, SPS) with the open Message Queue Telemetry Transport (MQTT) protocol. MQTT is a simple, yet very performing and robust publish/subscribe message passing system, which can also serve for event brokering as described in the SWE Service Model. Actual addressing and data transmission is done by a topic string and an arbitrary payload. The topic string and the payload need to reflect the necessary standard semantics to be mapped back and forth to allow for a seamless, and preferably lossless bi-directional multi-lateral communication between the WSN as data provider (SOS), web clients as data consumers (SOS) and the management system, which interacts with the WSN in a standardised way, too (SOS and SPS). The proposed architecture is illustrated in figure 2.

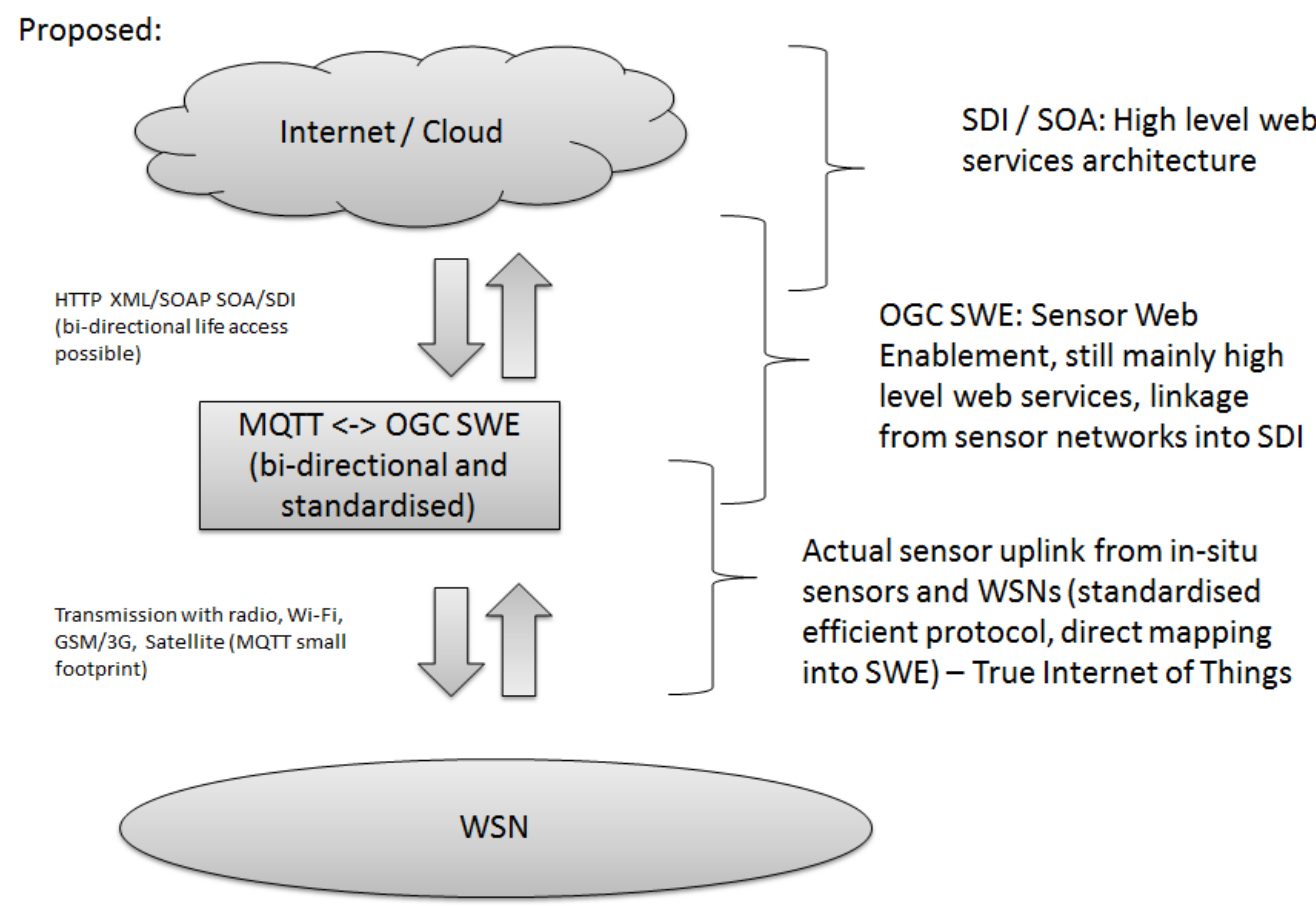

Figure 2: Proposed architecture with the MQTT-SWE proxy layer

We developed a mapping of the complex and generic SWE semantics within our MQTT implementation. The MQTT topic string per definition is an arbitrary UTF-8 encoded string with an overall size limit of 65535 bytes. The topic name at the time of publishing must not contain wildcard character (i.e. '*'), however subscribing to topics can make use of wildcard character. Furthermore, '/' can be used to structure topic name hierarchies. SOS and SPS define many operations, messages, which typically follow the request and response pattern, and the respective message encodings. For our experiment we only implemented a commonly used subset.

Table 1 lists operations and messages investigated for the proxy implementation. The full core profiles of OGC SOS and OGC SPS are handled through the SeNoMa framework within the cloud. The low-level sensor nodes are linked to the OGC-interfaces of the SeNoMa framework. The WSN itself does not need to implement all possible operations, but for the standards compliant integration of additional nodes, these should provide self-descriptive references, i.e. their specifications, monitoring outputs and tasking capabilities. The communication within the proxy layer happens through a standard off-the-shelf open source MQTT message queue application. The addressing via topic strings is crucial, as messaging within MQTT protocol allows only for three types of service quality: 1) send message once, 2) have message received exactly once by one client, 3) have message received at least once by one client. The message queue broker takes care of the passing and receiving of the messages. The QoS level 2 is used consistently. 
Ghobakhlou et al, Integration of Wireless Sensor Network and Web Services

Table 1: SWE-MQTT mapping (<actorID> is either sensorID or SeNoMa service)

\begin{tabular}{|c|c|c|c|}
\hline SWE Operation & SWE Encoding & MQTT Topic String & MQTT Payload \\
\hline $\begin{array}{l}\text { sos:InsertSensor } \\
\text { request }\end{array}$ & $\begin{array}{l}\text { Swes:InsertSensor containing } \\
\text { sml:System } \\
\text { text/xml;subtype="SensorML/1.0.1" }\end{array}$ & $\begin{array}{l}\text { sos/insertSensor/ } \\
\text { request/SeNoMa }\end{array}$ & $\begin{array}{l}\text { condensed binary } \\
\text { encoding of sensor } \\
\text { platform } \\
\text { characteristics }\end{array}$ \\
\hline $\begin{array}{l}\text { sos:InsertSensor } \\
\text { response }\end{array}$ & $\begin{array}{l}\text { swes:InsertSensorResponse } \\
\text { text/xml (acknowledge) }\end{array}$ & $\begin{array}{l}\text { sos/insertSensor/ } \\
\text { response/<sensorID> }\end{array}$ & $\begin{array}{l}\text { Acknowledge ok, } \\
\text { or reject with error }\end{array}$ \\
\hline $\begin{array}{l}\text { sos:InsertObservation } \\
\text { request }\end{array}$ & $\begin{array}{l}\text { sos:InsertObservation containing } \\
\text { om:Observation } \\
\text { text/xml;subtype="om/2.0.0" }\end{array}$ & $\begin{array}{l}\text { sos/insertObservation/ } \\
\text { request/SeNoMa }\end{array}$ & $\begin{array}{l}\text { condensed binary } \\
\text { encoding of } \\
\text { sensorID, } \\
\text { phenomenon and } \\
\text { measurement }\end{array}$ \\
\hline $\begin{array}{l}\text { sos:InsertObservation } \\
\text { response }\end{array}$ & $\begin{array}{l}\text { swes:InsertObservationResponse } \\
\text { text/xml (acknowledge) }\end{array}$ & $\mathrm{n} / \mathrm{a}$ & Not necessary \\
\hline $\begin{array}{l}\text { sos:GetObservation } \\
\text { request }\end{array}$ & $\begin{array}{l}\text { sos:GetObservation containing sos: } \\
\text { ogc: and fes: query parametrisation, } \\
\text { text/xml }\end{array}$ & $\begin{array}{l}\text { sos/getObservation/re } \\
\text { quest } /<\text { actorID> }\end{array}$ & $\begin{array}{l}\text { Condensed binary } \\
\text { encoding } \\
\text { requesting next } \\
\text { possible } \\
\text { observation of } \\
\text { <actorID> }\end{array}$ \\
\hline $\begin{array}{l}\text { sos:GetObservation } \\
\text { response }\end{array}$ & $\begin{array}{l}\text { sos:GetObservationResponse } \\
\text { containing om:Observation } \\
\text { text/xml;subtype="om/2.0.0" }\end{array}$ & $\begin{array}{l}\text { sos/getObservation/res } \\
\text { ponse/<reqActorID> }\end{array}$ & $\begin{array}{l}\text { Condensed binary } \\
\text { encoding of } \\
\text { observation to } \\
\text { requesting } \\
\text { <actorID> }\end{array}$ \\
\hline $\begin{array}{l}\text { swes:DescribeSensor } \\
\text { request }\end{array}$ & $\begin{array}{l}\text { swes:DescribeSensor containing } \\
\text { requested sensorID text/xml }\end{array}$ & $\begin{array}{l}\text { swes/describeSensor/ } \\
\text { request } /<\text { sensorID }>\end{array}$ & $\begin{array}{l}\text { Requested } \\
<\text { sensorID> }\end{array}$ \\
\hline $\begin{array}{l}\text { swes:DescribeSensor } \\
\text { response }\end{array}$ & $\begin{array}{l}\text { swes:DescribeSensorResponse } \\
\text { containing sml:System } \\
\text { text/xml;subtype="SensorML/1.0.1" }\end{array}$ & $\begin{array}{l}\text { swes/describeSensor/ } \\
\text { response/<reqActorID } \\
>\end{array}$ & $\begin{array}{l}\text { condensed binary } \\
\text { encoding of sensor } \\
\text { platform } \\
\text { characteristics }\end{array}$ \\
\hline $\begin{array}{l}\text { sps:DescribeTasking } \\
\text { request }\end{array}$ & $\begin{array}{l}\text { sps:DescribeTasking (generic } \\
\text { enquiry or containing particular } \\
\text { process) text/xml }\end{array}$ & $\begin{array}{l}\text { sps/describeTasking/ } \\
\text { request/<sensorID> }\end{array}$ & $\begin{array}{l}\text { Requested } \\
<\text { sensorID> }\end{array}$ \\
\hline $\begin{array}{l}\text { sps:DescribeTasking } \\
\text { response }\end{array}$ & $\begin{array}{l}\text { sps:DescribeTasking containing } \\
\text { om:Process and swe:DataRecord } \\
\text { elements with parameter description } \\
\text { for available tasking submission } \\
\text { text/xml }\end{array}$ & $\begin{array}{l}\text { sps/describeTasking/ } \\
\text { response/<reqActorID } \\
>\end{array}$ & $\begin{array}{l}\text { condensed binary } \\
\text { encoding of } \\
\text { assignable sensor } \\
\text { capabilities }\end{array}$ \\
\hline sps:Submit request & $\begin{array}{l}\text { sps:Submit containing om:Process } \\
\text { and swe:DataRecord elements with } \\
\text { actual parametrisation to initiate a } \\
\text { task text/xml }\end{array}$ & $\begin{array}{l}\text { sps/submit/ } \\
\text { request/<sensorID> }\end{array}$ & $\begin{array}{l}\text { condensed binary } \\
\text { encoding of } \\
\text { assignable sensor } \\
\text { capabilities }\end{array}$ \\
\hline sps:Submit response & $\begin{array}{l}\text { sps:SubmitResponse (acknowledge } \\
\text { of initialization of task containing } \\
\text { assigned unique taskID) text/xml }\end{array}$ & $\begin{array}{l}\text { sps/submit/ } \\
\text { response/<reqActorID } \\
>\end{array}$ & $\begin{array}{l}\text { Acknowledge ok } \\
\text { and assigned } \\
\text { unique taskID, or } \\
\text { error }\end{array}$ \\
\hline sps:GetStatus request & sps:GetStatus containing assigned & sps/getStatus/request/ & taskID and \\
\hline
\end{tabular}




\begin{tabular}{|l|l|l|l|}
\hline & taskID text/xml & <taskID> & reqActorID \\
\hline $\begin{array}{l}\text { sps:GetStatus } \\
\text { response }\end{array}$ & $\begin{array}{l}\text { sps:GetStatusResponse containing } \\
\text { percentage done and add. status info } \\
\text { for assigned taskID text/xml }\end{array}$ & $\begin{array}{l}\text { sps/getStatus/response } \\
/<\text { taskID }>/<\text { reqActorI } \\
\mathrm{D}>\end{array}$ & $\begin{array}{l}\text { condensed binary } \\
\text { encoding of } \\
\text { percentage done } \\
\text { and add. status info }\end{array}$ \\
\hline
\end{tabular}

\section{RESULTS}

The system architecture mentioned in Section 2.1 has been deployed in various locations as part of international collaboration initiative to micro-environment data monitoring and modelling for applications in agriculture. So far, networks have been installed in locations chosen for this research in Chile, Uruguay, India, Japan and New Zealand. All sensor data from these locations are uploaded to a remote central server.

A web application was developed to enable monitoring and visualization of climate, atmosphere, plants and soil data from each vineyard. The server side processes the incoming data and populates the database. Figure 3 depicts real-time streaming of temperature and humidity using the proposed OGC-SOS described in Section 4.
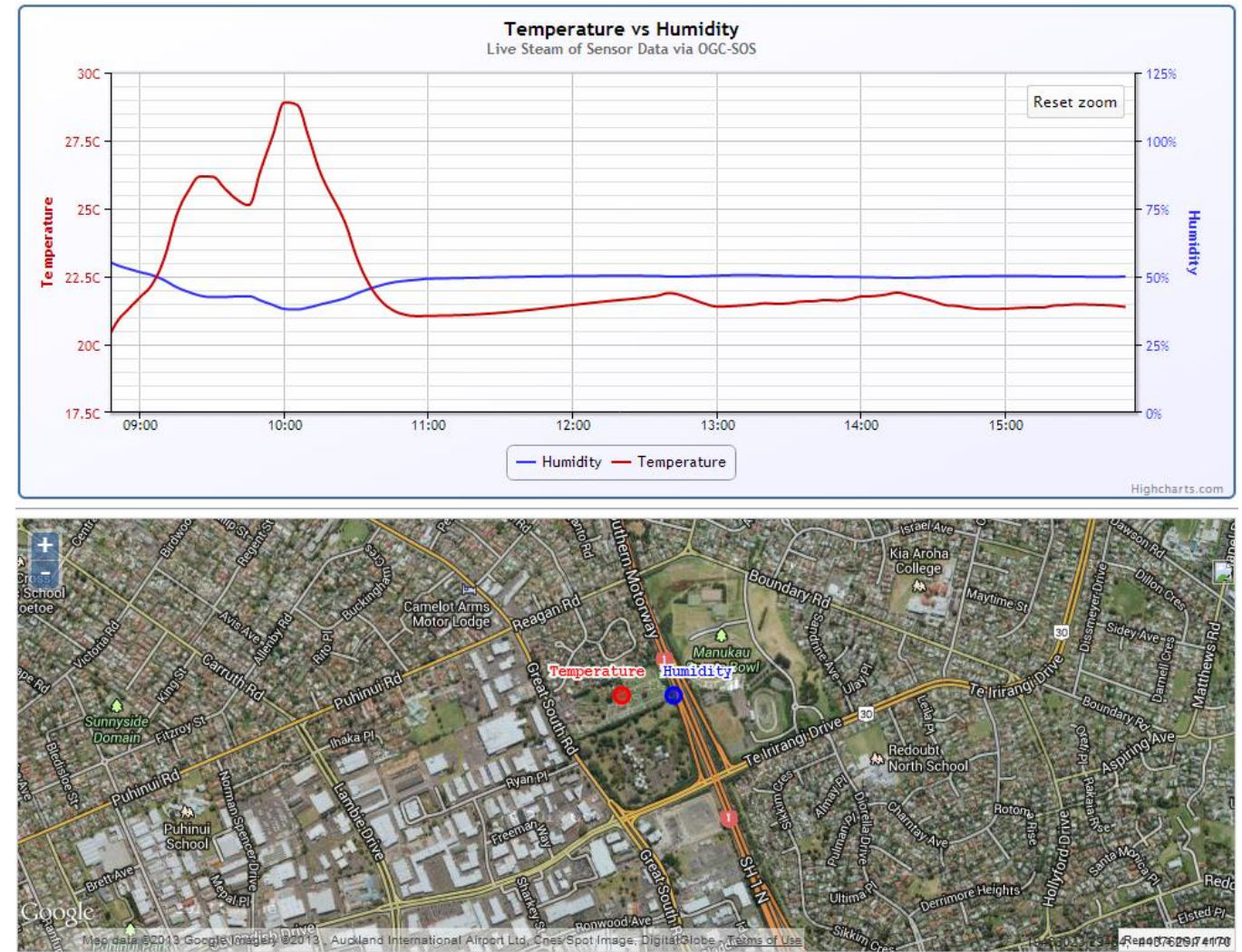

Figure 3: Illustration of a real-time streaming of Temperature and Humidity data using the proposed OGCSOS

The implementation of the asynchronous publish/subscribe MQTT protocol as message carrier platform equates well to the sleeping routines embedded in WSNs to preserve energy. The MQTT broker service ensures the proper queuing and delivery of requests and responses to and from the sensor platforms within their awake and sleep cycles.

\section{DISCUSSION AND FUTURE WORK}

The real-time monitoring system and web-based information system are designed for use by decision makers. With the leveraging of the established OGC Sensor Web Enablement standards and the reliability, performance, efficiency and robustness of the MQTT protocol, Wireless Sensor Networks can now be linked directly to ubiquitous SDI and SOA architectures for online data access and sensor configuration. The 
prposed framework has been seen as an improvement to the monitoring system through the application of SWE framework.

Futher work is required to create web-enabled remote management platform for the WSN. This will enable to configure and manage wireless sensors network through a simple Data Management API. The SPS implmentation described in this paper needs to be tested in real-world field installation replacing the existing deployment.

\section{REFERENCES}

BOTTS, M., PERCIVALL, G., REED, C. \& DAVIDSON, J. 2008. OGC® Sensor Web Enablement: Overview and High Level Architecture. In: NITTEL, S., LABRINIDIS, A. \& STEFANIDIS, A. (eds.) GeoSensor Networks. Springer Berlin Heidelberg.

BRÖRING, A., ECHTERHOFF, J., JIRKA, S., SIMONIS, I., EVERDING, T., STASCH, C., LIANG, S. \& LEMMENS, R. 2011. New Generation Sensor Web Enablement. Sensors, 11, 2652-2699.

CHEN, J., DÍAZ, M., RUBIO, B. \& TROYA, J. M. 2013. PS-QUASAR: A publish/subscribe QoS aware middleware for Wireless Sensor and Actor Networks. Journal of Systems and Software, 86, 1650-1662.

Deborah, E., Ramesh,G., John, H. and Satish, K.(1999) . Next century challenges: scalable coordination in sensor networks. In Proceedings of the 5th annual ACM/IEEE international conference on Mobile computing and networking Seattle, Washington, United States: ACM Press, pp. 263-270.

Díaz. S.E., Pérez J.C., Mateos A.C., Marinescu M.C. and Guerra B.B., 2011. A novel methodology for the monitoring of the agricultural production process based on wireless sensor networks. Computers and Electronics in Agriculture, 76, pp. 252-265.

ERICKSON, J. \& SIAU, K. 2008. Web Services, Service-Oriented Computing, and Service-Oriented Architecture: Separating Hype from Reality. Journal of Database Management, 19 42-54.

GRANELL, C., DÍAZ, L. \& GOULD, M. 2010. Service-oriented applications for environmental models: Reusable geospatial services. Environmental Modelling \& Software, 25, 182-198.

Ghobakhlou, A., Zandi, S. and Sallis, P. (2010) Development of Environmental Monitoring System with Wireless Sensor Networks. In 19th International Conference on Modelling and Simulation (MODSIM 2011) 12 - 16 December 2011(pp 1125-1131)

Ghobakhlou A., Perera A., Sallis P. and Zandi S. (2010). Modular sensor nodes for environmental data monitoring. Proceedings of the Fourth International Conference on Sensing Technology, University of Salento, Lecce, Italy June 3 - 5, 2010, pp 372-377 ISBN 978-0-473-16942-8

HUNKELER, U., LOMBRISER, C., TRUONG, H. L. \& WEISS, B. 2013. A case for centrally controlled wireless sensor networks. Computer Networks, 57, 1425-1442.

OGC 2007. Sensor Model Language (SensorML) Implementation Specification. 1.0.0. http://www.opengeospatial.org/standards/sensorml: The Open Geospatial Consortium (OGC).

OGC 2011a. Observations and Measurements - XML Implementation (O\&M v2.0 conceptual model also published as ISO/DIS 19156), O\&M v2.0. O\&M 2.0. http://www.opengeospatial.org/standards/is: The Open Geospatial Consortium (OGC).

OGC 2011b. OGC SWE Common Data Model Encoding Standard, v2.0. SWE 2.0. http://www.opengeospatial.org/standards/is: The Open Geospatial Consortium (OGC).

OGC 2012. OGC Sensor Observation Service Interface Standard, v2.0. SOS 2.0. http://www.opengeospatial.org/standards/is: The Open Geospatial Consortium (OGC).

OGC 2013. OGC Standards. http://www.opengeospatial.org/standards: Open Geospatial Consortium.

Zhang, R., Guo, J., Zhang, L., Zhang, Y., Wang, L. and Wang, Q., 2011. A calibration method of detecting soil water content based on the information-sharing in wireless sensor network. Computers and Electronics in Agriculture, 76, pp. 161-168. 